\title{
Irrationale Dezimalzahlen
}

\author{
Günter Köhler and Jürgen Spilker
}

\begin{abstract}
Günter Köhler ist seit 1976 Professor an der Universität Würzburg. Jürgen Spilker ist pensionierter Professor an der Universität Freiburg (Breisgau). Die beiden Autoren verbindet nicht nur ihr Alter von 64 bzw. 68 Jahren, sondern auch die Freude an Problemen und Ideen aus der elementaren Zahlentheorie und Analysis.
\end{abstract}

\section{Einleitung}

Die rationalen Zahlen kann man mit den natürlichen Zahlen durchnummerieren, die irrationalen nicht. Unter den reellen Zahlen gibt es mehr irrationale als rationale. Trotzdem begegnen uns im täglichen Leben eher rationale als irrationale Zahlen. Ein Grund dafür ist, dass die Rationalität einer reellen Zahl meist evident ist, die Irrationalität dagegen eines Beweises bedarf. Es ist deshalb sinnvoll, explizite Beispiele für irrationale Zahlen anzugeben. In dieser Note geschieht das mittels Dezimalzahlen.

Es sei $A=\left(a_{n}\right)_{n \geq 1}$ eine Folge von natürlichen Zahlen, die wir uns als Dezimalzahlen vorgeben. Schreibt man die Ziffern von $a_{1}$ nacheinander hin, beginnend mit $0, * * \ldots$, hängt die von $a_{2}$ an, sodann die von $a_{3}$ usw., dann erhält man eine reelle Zahl aus dem Einheitsintervall, die wir mit $r_{A}$ bezeichnen. Diese Dezimalzahl ist genau dann irrational, wenn sie weder abbricht noch schließlich periodisch ist. Das ist zum Beispiel dann der Fall, wenn es zu jeder natürlichen Zahl $k$ mindestens zwei verschiedene Folgenglieder mit genau $k$ Ziffern gibt. Darauf basiert unser erstes Irrationalitäts-Kriterium (Satz 1). Andererseits ist das auch der Fall, wenn in $r_{A}$ beliebig lange Blöcke der Ziffer 0 auftreten. Darauf basieren die anderen Kriterien (Sätze 2, 3 und 4). In Satz 3 wird aus dem

Obwohl es mehr irrationale als rationale Zahlen gibt, ist es schwieriger, irrationale Zahlen explizit zu benennen. In dieser Arbeit werden mit vier Methoden Dezimalzahlen konstruiert, welche irrational sind. Dazu werden die Ziffern der Glieder von geeigneten Folgen nacheinander hingeschrieben. Einfache Beispiele dazu sind
$0,14916253649 \ldots$
(Quadrate),
$0,12624120720 \ldots$
(Fakultäten),
$0,11121133114641 \ldots$
(Potenzen von 11),
$0,112358132134 \ldots$
(Fibonacci). 
asymptotischen Verhalten der Folge $\left(a_{n}\right)_{n \geq 1}$ geschlossen, dass es große Nullenblöcke gibt. Der vierte Satz behandelt Lösungen von linearen Rekurrenzen. Wenn eine Nullstelle des Begleitpolynoms die anderen dominiert, dann folgt die richtige Asymptotik, und Satz 3 ist anwendbar.

Obwohl die vier Irrationalitäts-Kriterien elementar zu beweisen sind, haben wir sie nicht in der Literatur gefunden. Beispiele zu den Sätzen enthält schon das klassische Zahlentheoriebuch von Hardy und Wright ([3], Theoreme 137 und 138). Wir geben viele weitere Beispiele an. Dazu gehören auch die Fibonacci- und die Lucas-Folge. Sie sind Inhalt der Aufgabe 1193 [4]. Diese hat den Anstoß zu dieser Arbeit gegeben.

Notation: Reelle Zahlen $x$ zerlegen wir in der Form $x=\lfloor x\rfloor+\{x\}$ mit $\lfloor x\rfloor \in \mathbb{Z}$ und $0 \leq\{x\}<1$. Wir zählen die Null nicht zu den natürlichen Zahlen.

\section{Das erste Kriterium}

Es sei $A=\left(a_{n}\right)_{n \geq 1}$ eine Folge von natürlichen Zahlen und

$$
\begin{aligned}
& a_{n}=a_{n 0} 10^{k(n)}+a_{n 1} 10^{k(n)-1}+\cdots+a_{n, k(n)}, \\
& a_{n j} \in\{0,1, \ldots, 9\} \quad \text { für alle } j, \quad a_{n 0} \neq 0,
\end{aligned}
$$

die Dezimaldarstellung von $a_{n}$. Wir betrachten die reelle Zahl

$$
r_{A}:=0, a_{10} a_{11} \ldots a_{1, k(1)} a_{20} a_{21} \ldots a_{2, k(2)} a_{30} \ldots
$$

Die folgenden vier Sätze enthalten hinreichende Kriterien für die Irrationalität der Dezimalzahl $r_{A}$. Dabei ist die Basis 10 willkürlich gewählt. Alle folgenden Aussagen gelten in analoger Weise auch für Darstellungen zu einer anderen Basis.

Satz 1 Die Folge $A=\left(a_{n}\right)_{n \geq 1}$ von natürlichen Zahlen habe folgende Eigenschaften:

$A$ ist schließlich schwach monoton steigend;

zu jedem $k \geq k_{0}$ existiert ein $n$, so dass $10^{k-1} \leq a_{n}<a_{n+1}<10^{k}$ gilt.

Dann ist $r_{A}$ irrational.

Beweis. Wir nehmen an, es gäbe so eine Folge $A$ mit rationalem $r_{A}$. Weil die Dezimalzahl $r_{A}$ nicht abbricht, ist sie schließlich periodisch ([3], Theorem 136). Es sei $s$ die Länge der Periode und $t$ die Länge der Vorperiode. Wegen (2) existieren natürliche Zahlen $q$ und $n$ mit $q s>t+1$ und $10^{q s-1} \leq a_{n}<a_{n+1}<10^{q s}$. Da $q s$ ebenfalls Länge einer Periode ist, stimmen die ersten Ziffern von $a_{n}$ und von $a_{n+1}$ überein, ebenso die zweiten usw. Also ist $a_{n}=a_{n+1}$, und das ist ein Widerspruch zur Wahl von $n$.

Beispiele zu Satz 1. Die Folge aller natürlichen Zahlen führt auf die irrationale Champernowne-Zahl ([2], S. 255)

$0,123456789101112 \ldots$ 
Man kann auch nur die Zahlen nehmen, die eine feste Ziffer nicht enthalten, zum Beispiel die 2 , also

$$
0,1345678910111314 \ldots
$$

Eine große Menge von Beispielen bekommt man durch die Eigenschaft

$$
a_{n}<a_{n+1} \leq 10 a_{n-1} \quad \text { für alle } \quad n>n_{0}
$$

denn zu jedem natürlichen $k$ existiert ein kleinstes $n$ mit $a_{n} \geq 10^{k-1}$, und $n$ ist größer als $n_{0}$, falls $k$ groß genug ist; dann folgt $a_{n+1} \leq 10 a_{n-1}<10^{k}$. Diese Klasse enthält

$$
0,23571113171923 \ldots
$$

denn für die Primzahlfolge $\left(p_{n}\right)_{n \geq 1}$ gilt $p_{n+1} \leq 2 p_{n}$ für alle $n$ (Bertrandsches Postulat: [3], Theorem 418). Fügt man in eine Folge mit der Eigenschaft (3) weitere Glieder ein, dann bleibt (3) erhalten. Also führt auch die Folge aller Primzahlpotenzen auf eine irrationale Zahl:

$$
0,2345789111316171923 \ldots
$$

Weitere Beispiele sind

$$
\begin{array}{ll}
0,46891012141516 \ldots & \text { (zusammengesetzte Zahlen), } \\
0,1491625364964 \ldots & \text { (Quadratzahlen), } \\
0,2356710111314 \ldots & \text { (quadratfreie Zahlen). }
\end{array}
$$

Auch die $l$-ten Potenzen $(l \geq 2)$, die Folge $a_{n}=\lfloor\alpha n\rfloor$ mit reellem $\alpha>0$ sowie $a_{n}=\lfloor n \log n\rfloor$ gehören in diese Klasse. Weitere Beispiele mit der Eigenschaft (3) sind die Fibonacci-Zahlen

$$
a_{1}=a_{2}=1, a_{n+2}=a_{n+1}+a_{n} \quad \text { für } \quad n \geq 1
$$

(auch der Satz 4 ist anwendbar) sowie die Tribonacci-Zahlen [6]

$$
a_{1}=a_{2}=1, a_{3}=2, a_{n+3}=a_{n+2}+a_{n+1}+a_{n} \quad \text { für } \quad n \geq 1 .
$$

Denn in beiden Fällen gilt $a_{n}<a_{n+1} \leq 2 a_{n}$ für alle $n>1$.

\section{Das zweite Kriterium}

Die nächsten drei Sätze basieren darauf, dass periodische Dezimalzahlen keine beliebig großen, endlichen Blöcke aus Nullen haben.

Satz 2 In der Folge $A=\left(a_{n}\right)_{n \geq 1}$ von natürlichen Zahlen existiere zu jeder natürlichen Zahl s ein Folgenglied $a_{n}$, das einen Block von mindestens s aufeinanderfolgenden Nullen in seiner Dezimaldarstellung hat. Dann ist $r_{A}$ irrational. 
Der Beweis ist trivial, denn die Dezimalzahl $r_{A}$ kann weder abbrechen noch schließlich periodisch sein. Sie ist also nicht rational.

Bemerkung. Die Ziffer 0 in Satz 2 ist willkürlich. Die Zahl $r_{A}$ ist auch dann irrational, wenn sie beliebig große Blöcke aus irgendeiner Ziffer $e$ enthält, aber nicht mit einem unendlichen Block eee... endet.

Ein Beispiel zu Satz 2 ist $a_{n}=n$ !; denn die Zahl $a_{n}$ mit $n \geq 10^{s}$ endet mit mehr als $s$, nämlich genau $\sum_{k \geq 1}\left\lfloor n / 5^{k}\right\rfloor$ Nullen. Ein anderes Beispiel ist die charakteristische Folge der Primzahlen,

$$
a_{n}= \begin{cases}1 & \text { falls } n \text { prim ist } \\ 0 & \text { sonst; }\end{cases}
$$

denn zwischen $s !+2$ und $s !+s$ liegt keine Primzahl. Dasselbe Resultat gilt für die charakteristische Folge der Primzahlpotenzen, und somit ist die Zahl

$$
0,01111011101010011010001 \ldots
$$

irrational. Denn für alle $s \geq 4$ liegt zwischen $s !+2$ und $s !+\lfloor\sqrt{s}\rfloor$ keine Primzahlpotenz.

\section{Das dritte Kriterium}

Aus dem asymptotischen Verhalten der Folge $A$ kann man manchmal schließen, dass es beliebig große Blöcke aus Nullen gibt.

Satz 3 Die Folge $A=\left(a_{n}\right)_{n \geq 1}$ von natürlichen Zahlen habe die Form

$$
a_{n} \sim \gamma \alpha^{n} \text { mit } \gamma, \alpha \in \mathbb{R}, \gamma>0, \alpha>1
$$

$\log \alpha$ ist irrational.

Dann ist $r_{A}$ irrational.

Bemerkung. In dieser Note haben alle Logarithmen die Basis 10.

Beweis des Satzes. Wegen (4) gilt

$$
a_{n}=\gamma \alpha^{n}\left(1+\rho_{n}\right) \quad \text { mit } \quad \lim _{n \rightarrow \infty} \rho_{n}=0 .
$$

Wir zeigen, dass man hierauf den Satz 2 anwenden kann. Dazu wählen wir eine natürliche Zahl $s_{0}$ mit

$$
\{-\log \gamma\}+\log \left(1+\frac{1}{10^{s_{0}}}\right)<1
$$

sowie ein festes $s \geq s_{0}$. Es existiert ein $n_{0}$ mit

$$
\begin{aligned}
\left|\rho_{n}\right| & \leq\left|\rho_{n_{0}}\right|<1, \\
\left|\log \left(1+\rho_{n}\right)\right| & <\frac{1}{3} \log \left(1+10^{-s}\right),
\end{aligned}
$$


für alle $n \geq n_{0}$. Wegen (5) ist die Folge $(n \log \alpha)_{n \geq 1}$ gleichverteilt modulo 1 ([5], S. 8). Also gibt es ein natürliches $n>\max \left\{n_{0},(s-\log \gamma) / \log \alpha\right\}$ mit

$$
\{-\log \gamma\}+\frac{1}{3} \log \left(1+10^{-s}\right)<\{n \log \alpha\}<\{-\log \gamma\}+\frac{2}{3} \log \left(1+10^{-s}\right) .
$$

Mittels (8) folgt hieraus

$$
0<\{n \log \alpha\}-\{-\log \gamma\}+\log \left(1+\rho_{n}\right)<\log \left(1+10^{-s}\right) .
$$

Mit $K:=\lfloor n \log \alpha\rfloor-\lfloor-\log \gamma\rfloor=\log a_{n}-\{n \log \alpha\}+\{-\log \gamma\}-\log \left(1+\rho_{n}\right)$ gilt also

$$
K<\log a_{n}<K+\log \left(1+10^{-s}\right)
$$

und

$$
10^{k}<a_{n}<10^{k}\left(1+10^{-s}\right) .
$$

Wegen $K \geq s$ hat dieses $a_{n}$ die Form $100 \ldots 0 * \ldots *$ mit einem Nullenblock der Länge $s$. Da das für alle großen $s$ gilt, ergibt Satz 2 die Behauptung.

Beispiele zu Satz 3 sind $a_{n}=\gamma \alpha^{n}$ mit $\gamma, \alpha \in \mathbb{N}, \alpha \neq 10^{k}$ für alle $k \geq 0$. Weitere Beispiele folgen im nächsten Abschnitt.

Wir verallgemeinern Satz 3 zu

Satz $3^{\prime}$ Hat die Folge $A=\left(a_{n}\right)_{n \geq 1}$ die Eigenschaften

$$
a_{n} \sim \gamma n^{m} \alpha^{n} \text { mit } \gamma, \alpha \in \mathbb{R}, \gamma>0, \alpha>1, m \in \mathbb{N}_{0},
$$

und ist $\log \alpha$ irrational, dann ist $r_{A}$ irrational.

Der Beweis verläuft wie der von Satz 3. Man ersetzt die Folge $(n \log \alpha)_{n \geq 1}$ durch $(m \log n+n \log \alpha)_{n \geq 1}$, welche nach [5], Theorem 3.3, S. 28, ebenfalls gleichverteilt modulo 1 ist.

\section{Anwendung auf rekurrente Folgen}

Wir betrachten eine lineare Rekursion $k$-ten Grades $(k \geq 2)$ :

$$
a_{n+k}=c_{1} a_{n+k-1}+c_{2} a_{n+k-2}+\cdots+c_{k} a_{n}, \quad n \geq 1,
$$

mit ganzzahligen Koeffizienten $c_{1}, c_{2}, \ldots, c_{k}, c_{k} \neq 0$, und Anfangswerten $a_{1}, a_{2}, \ldots$, $a_{k} \in \mathbb{N}$. Wir nehmen an, dass die rekursiv definierte Folge $A=\left(a_{n}\right)_{n \geq 1}$ aus natürlichen Zahlen besteht. Das Begleitpolynom zerfällt im Komplexen, etwa

$$
X^{k}-c_{1} X^{k-1}-\cdots-c_{k}=\prod_{j=1}^{k}\left(X-\alpha_{j}\right), \quad \alpha_{j} \in \mathbb{C} .
$$

Wir wollen Satz 3 anwenden und setzen deshalb voraus, dass eine Nullstelle, etwa $\alpha_{1}$, dominiert und der Logarithmus ihres Betrages irrational ist. 
Satz 4 Wenn

$$
\left|\alpha_{1}\right|>\left|\alpha_{j}\right| \quad \text { für } \quad 2 \leq j \leq k
$$

und

$$
\log \left|\alpha_{1}\right| \quad \text { irrational }
$$

ist, dann ist auch $r_{A}$ irrational.

Beweis. Nach der Formel von Binet ([1], Satz 3.1, S. 61) existieren eine komplexe Zahl $\beta \neq 0$ und komplexe Polynome $p_{j}(X), 2 \leq j \leq k$, mit

$$
a_{n}=\beta \alpha_{1}^{n}+\sum_{j=2}^{k} p_{j}(n) \alpha_{j}^{n}=|\beta|\left|\alpha_{1}\right|^{n}\left(1+\rho_{n}\right), \quad \lim _{n \rightarrow \infty} \rho_{n}=0 .
$$

Also gelten (4) und (5) mit $\gamma=|\beta|>0, \alpha=\left|\alpha_{1}\right|>1$, und Satz 3 ergibt die Behauptung.

Bemerkung. In der Voraussetzung von Satz 4 kommen die Anfangsglieder $a_{1}, a_{2}, \ldots, a_{k}$ nicht vor.

Die Voraussetzung (10) darf man nicht weglassen: Die Rekursion

$$
a_{1}=2, a_{2}=22, a_{n+2}=11 a_{n+1}-10 a_{n} \quad \text { für } n \geq 1
$$

hat die Lösung $a_{n}=\frac{2}{9}\left(10^{n}-1\right)$, und es ist $r_{A}=\frac{2}{9}, \alpha_{1}=10$.

Beispiele zu Satz 4 sind die Fibonacci-Folge $a_{1}=a_{2}=1, a_{n+2}=a_{n+1}+a_{n}(n \geq 1)$, und die Lucas-Folge $a_{1}=1, a_{2}=3, a_{n+2}=a_{n+1}+a_{n}(n \geq 1)$; denn der Logarithmus von $\alpha_{1}=\frac{1+\sqrt{5}}{2}$ ist irrational.

Wir schließen mit einer Verallgemeinerung von Satz 4.

Satz 4' Wenn

$$
\alpha_{1}=\alpha_{2}=\cdots=\alpha_{l} \quad \text { für ein } l \text { und } \quad\left|\alpha_{1}\right|>\left|\alpha_{j}\right| \text { für } l<j \leq k
$$

ist und (10) gilt, dann ist $r_{A}$ irrational.

Beweis. Es gilt $a_{n}=p_{1}(n) \alpha_{1}^{n}+\sum_{j=l+1}^{k} p_{j}(n) \alpha_{j}^{n}$ mit einem komplexen Polynom $p_{1} \neq 0$ vom Grad $m<l$ und irgendwelchen Polynomen $p_{l+1}, \ldots, p_{k}$ ([1], Satz 3.1, S. 61). Also folgt $a_{n} \sim|\beta| n^{m}\left|\alpha_{1}\right|^{n}$, und Satz $3^{\prime}$ ergibt die Behauptung.

Ein Beispiel mit $\alpha_{1}=\alpha_{2}$ ist

$$
a_{1}=2, a_{2}=8, a_{n+2}=4 a_{n+1}-4 a_{n} \quad \text { für } \quad n \geq 1 .
$$

Die zugehörige Folge lautet $a_{n}=n 2^{n}$. 


\section{Literatur}

[1] Aigner, M.: Diskrete Mathematik. Vieweg Verlag, Braunschweig/Wiesbaden 1996.

[2] Champernowne, D.G.: The construction of decimals normal in the base of ten. J. London Math. Soc. 8 (1933), 254-260.

[3] Hardy, G.H.; Wright, E.M.: An Introduction to the Theory of Numbers. Clarendon Press, Oxford 1954.

[4] Herrmann, E.: Aufgabe 1193. Elem. Math. 58 (2003), 84.

[5] Kuipers, L.; Niederreiter, H.: Uniform Distribution of Sequences. John Wiley, New York 1974.

[6] Scott, A.; Delaney, T.; Hoggatt, V.E.: The Tribonacci Sequence. Fibonacci Quart. 15 (1977), 193-200.

Günter Köhler

Universität Würzburg

Mathematisches Institut

Am Hubland

D-97074 Würzburg, Deutschland

e-mail: koehler@mathematik. uni-wuerzburg.de

Jürgen Spilker

Albert-Ludwigs-Universität Freiburg

Mathematisches Institut

Eckerstr. 1

D-79104 Freiburg, Deutschland

e-mail: spilker@mathematik. uni-freiburg.de 